\title{
Highly oscillatory quadrature
}

\author{
Daan Huybrechs and Sheehan Olver
}

\begin{abstract}
Oscillatory integrals are present in many applications, and their numerical approximation is the subject of this paper. Contrary to popular belief, their computation can be achieved efficiently, and in fact, the more oscillatory the integral, the more accurate the approximation. We review several existing methods, including the asymptotic expansion, Filon method, Levin collocation method and numerical steepest descent. We also present recent developments for each method.
\end{abstract}

\subsection{Introduction}

The aim of this paper is to review recent methods for the evaluation of the oscillatory integral

$$
I[f]=\int_{\Omega} f(\boldsymbol{x}) \mathrm{e}^{\mathrm{i} \omega g(\boldsymbol{x})} \mathrm{d} x,
$$

where $f$ and $g$ are nonoscillatory functions, the frequency of oscillations $\omega$ is large and $\Omega$ is some piecewise smooth domain. By taking the real and imaginary parts of this integral, we obtain integrals with trigonometric kernels:

$$
\Re I[f]=\int_{\Omega} f(\boldsymbol{x}) \cos \omega g(\boldsymbol{x}) \mathrm{d} V \quad \text { and } \quad \Im I[f]=\int_{\Omega} f(\boldsymbol{x}) \sin \omega g(\boldsymbol{x}) \mathrm{d} V .
$$

Highly oscillatory integrals of this form play a valuable role in applications. Using the modified Magnus expansion [17], highly oscillatory differential equations of the form $y^{\prime \prime}+g(t) y=0$, where $g(t) \rightarrow \infty$ while the derivatives of $g$ are moderate, can be expressed in terms of an infinite sum of highly oscillatory integrals. Differential equations of this form 
appear in many areas, including special functions, e.g., the Airy function. Oscillatory integrals also typically appear in scientific disciplines that involve the modelling of wave phenomena. For example in acoustics, the boundary element method requires the evaluation of highly oscillatory integrals, in order to solve integral equations with oscillatory kernels [16]. Modified Fourier series use highly oscillatory integrals to obtain a function approximation scheme that converges faster than the standard Fourier series ([20], reviewed in [13]). There are many other applications, including fluid dynamics, image analysis and more.

To understand why we need special methods for oscillatory integrals, it is important to study where traditional quadrature methods fail. Most nonoscillatory quadrature methods approximate an integral by a weighted sum sampling the integrand at $n$ discrete points $\left\{x_{1}, \ldots, x_{n}\right\}$, and averaging the samples with suitable weights $\left\{w_{1}, \ldots, w_{n}\right\}$ :

$$
\int_{a}^{b} w(x) f(x) \mathrm{d} x \approx \sum_{k=1}^{n} w_{k} f\left(x_{k}\right),
$$

where $w$ is some nonnegative weight function. Regardless of the particular method used, (1.1) fails as a quadrature scheme for high frequency oscillation when $w(x) \equiv 1$, unless $n$ grows with $\omega$. To see this, consider the integral

$$
\int_{a}^{b} f(x) \mathrm{e}^{\mathrm{i} \omega x} \mathrm{~d} x \approx \sum_{k=1}^{n} w_{k} f\left(x_{k}\right) \mathrm{e}^{\mathrm{i} \omega x_{k}},
$$

where $n, w_{k}$ and $x_{k}$ are all fixed for increasing $\omega$. Assuming that this sum is not identically zero, it cannot decay as $\omega$ increases. This can be seen in Figure 1.1, for the integral

$$
\int_{0}^{1} x^{2} \mathrm{e}^{\mathrm{i} \omega x} \mathrm{~d} x .
$$

A simple application of integration by parts - which will be investigated further in the next section-reveals that the integral itself decays like $\mathcal{O}\left(\omega^{-1}\right)$. Thus the error of any weighted sum is $\mathcal{O}(1)$, which compares to an error of order $\mathcal{O}\left(\omega^{-1}\right)$ when approximating the integral by zero! It is safe to assume that a numerical method which is less accurate than equating the integral to zero is of little practical use. On the other hand, letting $n$ be proportional to the frequency can result in considerable computational costs. This is magnified significantly when we attempt to integrate over multivariate domains. Even nonoscillatory quadrature is computationally difficult for multivariate integrals, and high oscillations 

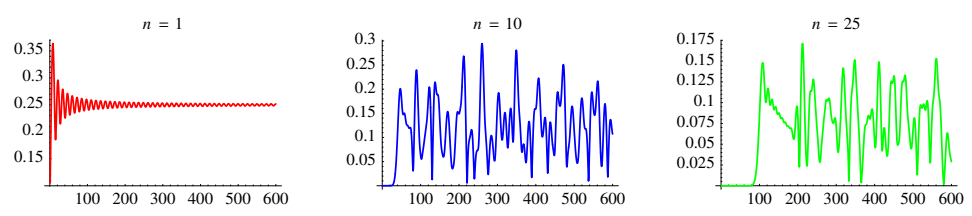

Fig. 1.1. The absolute error in approximating $\int_{0}^{1} x^{2} \mathrm{e}^{\mathrm{i} \omega x} \mathrm{~d} x$ as a function of $\omega$ by an $n$-point Gauss-Legendre quadrature scheme, for $n=1,10$ and 25 .

would only serve to further exasperate the situation. Thus we must look for alternative methods to approximate such integrals.

In this paper, we focus on four methods of approximation: the asymptotic expansion, Filon method, Levin collocation method and numerical steepest descent. An enormous amount of research has been conducted on the asymptotics of oscillatory integrals, hence we investigate the derivation of an asymptotic expansion in Section 1.2. Here there is a distinction between integrals with stationary points - points where $g^{\prime}(x)$ vanishes - and those without. We can also derive asymptotic results for multivariate integrals.

With the asymptotic groundwork in place, we consider in depth methods based on the Filon method in Section 1.3. These include Filontype methods which achieve higher asymptotic orders and Moment-free Filon-type methods for irregular oscillators with stationary points. We can also derive a multivariate Filon-type method, though only for linear oscillators over a simplex.

Like Moment-free Filon-type methods, Levin collocation methods of Section 1.4 can be constructed for irregular oscillators, though only for oscillators without stationary points. We also develop Levin-type methods which obtain higher asymptotic orders. Alternatively, we can use a Chung, Evans and Webster construction which frames the method in a more traditional quadrature context. Levin-type methods' generality lends itself to multivariate integration, which can be accomplished over more general domains than Filon-type methods, as long as a nonresonance condition is satisfied.

Steepest descent is a classical method for determining the asymptotic expansion of oscillatory integrals with stationary points. Recently, it has been used as a numerical quadrature scheme, which is reviewed in Section 1.5. These methods achieve twice the asymptotic decay for the same amount of function evaluations as Filon-type and Levin-type 
methods. They are also applicable to multivariate integrals, even to those which do not satisfy the nonresonance condition.

Finally, we give a brief overview of other existing methods in Section 1.6, for both Fourier oscillators and irregular oscillators. The methods for Fourier oscillators are generally based on standard quadrature methods, so the asymptotic order achievable is limited to either $\mathcal{O}\left(\omega^{-1}\right)$ or, with the use of endpoint data, $\mathcal{O}\left(\omega^{-2}\right)$.

\subsection{Asymptotics}

Whereas standard quadrature schemes are inefficient, a straightforward alternative exists in the form of asymptotic expansions. Asymptotic expansions actually improve with accuracy as the frequency increases, and - assuming sufficient differentiability of $f$ and $g$ - to arbitrarily high order. Furthermore the number of operations required to produce such an expansion is independent of the frequency, and extraordinarily small. Even more surprising is that this is all obtained by only requiring knowledge of the function at very few critical points within the interval - the endpoints and stationary points - as well as its derivatives at these points if higher asymptotic orders are required. There is, however, one critical flaw which impedes their use as quadrature formulæ: asymptotic expansions do not in general converge when the frequency is fixed, hence their accuracy is limited.

\subsubsection{Asymptotic expansion for integrals without stationary points}

Whenever $g$ is free of stationary points-i.e., $g^{\prime}(x) \neq 0$ within the interval of integration - we can derive an asymptotic expansion in a very straightforward manner by repeatedly applying integration by parts. The first term of the expansion is determined as follows:

$$
\begin{aligned}
I[f] & =\int_{a}^{b} f(x) \mathrm{e}^{\mathrm{i} \omega g(x)} \mathrm{d} x=\frac{1}{\mathrm{i} \omega} \int_{a}^{b} \frac{f(x)}{g^{\prime}(x)} \frac{\mathrm{d}}{\mathrm{d} x} \mathrm{e}^{\mathrm{i} \omega g(x)} \mathrm{d} x \\
& =\frac{1}{\mathrm{i} \omega}\left[\frac{f(b)}{g^{\prime}(b)} \mathrm{e}^{\mathrm{i} \omega g(b)}-\frac{f(a)}{g^{\prime}(a)} \mathrm{e}^{\mathrm{i} \omega g(a)}\right]-\frac{1}{\mathrm{i} \omega} \int_{a}^{b} \frac{\mathrm{d}}{\mathrm{d} x}\left[\frac{f(x)}{g^{\prime}(x)}\right] \mathrm{e}^{\mathrm{i} \omega g(x)} \mathrm{d} x .
\end{aligned}
$$

The term

$$
\frac{1}{\mathrm{i} \omega}\left[\frac{f(b)}{g^{\prime}(b)} \mathrm{e}^{\mathrm{i} \omega g(b)}-\frac{f(a)}{g^{\prime}(a)} \mathrm{e}^{\mathrm{i} \omega g(a)}\right]
$$




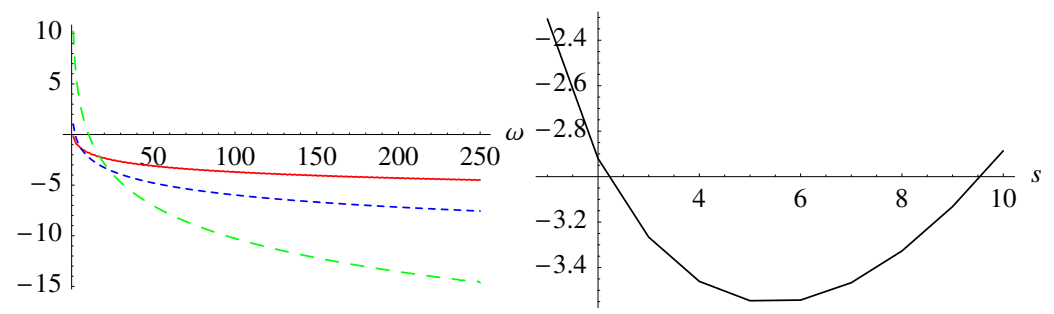

Fig. 1.2. The base-10 logarithm of the error in approximating $\int_{0}^{1} \cos x \mathrm{e}^{\mathrm{i} \omega\left(x^{2}+x\right)} \mathrm{d} x$. The left graph compares the one-term (solid line), three-term (dotted line) and ten-term (dashed line) asymptotic expansions. The right graph shows the error in the $s$-term asymptotic expansion for $\omega=20$.

approximates the integral $I[f]$ with an error

$$
-\frac{1}{\mathrm{i} \omega} I\left[\frac{\mathrm{d}}{\mathrm{d} x}\left[\frac{f(x)}{g^{\prime}(x)}\right]\right]=\mathcal{O}\left(\omega^{-2}\right)
$$

using the fact that the integral decays like $\mathcal{O}\left(\omega^{-1}\right)$ [35]. Thus the more oscillatory the integrand, the more accurately (1.2) can approximate the integral, with a relative accuracy $\mathcal{O}\left(\omega^{-1}\right)$. Moreover the error term is itself an oscillatory integral, thus we can integrate by parts again to obtain an approximation with an error $\mathcal{O}\left(\omega^{-2}\right)$. Iterating this procedure results in an asymptotic expansion:

Theorem 1 Suppose that $g^{\prime} \neq 0$ in $[a, b]$. Then

$$
I[f] \sim-\sum_{k=1}^{\infty} \frac{1}{(-\mathrm{i} \omega)^{k}}\left\{\sigma_{k}(b) \mathrm{e}^{\mathrm{i} \omega g(b)}-\sigma_{k}(a) \mathrm{e}^{\mathrm{i} \omega g(a)}\right\},
$$

where

$$
\sigma_{1}=\frac{f}{g^{\prime}}, \quad \sigma_{k+1}=\frac{\sigma_{k}^{\prime}}{g^{\prime}}, \quad k \geq 1
$$

We can find the error term for approximating $I[f]$ by the first $s$ terms of this expansion:

$$
I[f]=-\sum_{k=1}^{s} \frac{1}{(-\mathrm{i} \omega)^{k}}\left\{\sigma_{k}(b) \mathrm{e}^{\mathrm{i} \omega g(b)}-\sigma_{k}(a) \mathrm{e}^{\mathrm{i} \omega g(a)}\right\}+\frac{1}{(-\mathrm{i} \omega)^{s}} I\left[\sigma_{s}^{\prime}\right]
$$


In Figure 1.2 we use the partial sums of the asymptotic expansion to approximate the integral

$$
\int_{0}^{1} \cos x \mathrm{e}^{\mathrm{i} \omega\left(x^{2}+x\right)} \mathrm{d} x
$$

We compare three partial sums of the asymptotic expansion in the left graph: $s$ equal to one, three and ten. This graph demonstrates that increasing the number of terms used in the expansion does indeed increase the rate that the error in approximation goes to zero for increasing $\omega$. However, for any given frequency the expansion reaches an optimal error, after which adding terms to the expansion actually increases the error. This is shown in the right graph for $\omega$ fixed to be 20 , in which case the optimal expansion consists of five terms.

\subsubsection{Asymptotic expansions in the presence of stationary points}

Consider the following integral with a single stationary point:

$$
\int_{a}^{b} f(x) \mathrm{e}^{\mathrm{i} \omega g(x)} \mathrm{d} x
$$

where, for $\xi \in(a, b), 0=g(\xi)=g^{\prime}(\xi)=\cdots=g^{(r-1)}(\xi), g^{(r)}(\xi)>0$ and $g^{\prime}(x) \neq 0$ for $x \in[a, b] \backslash \xi$. If $g(\xi) \neq 0$, the integral can easily by written in this form by replacing $g$ by $g-g(\xi)$ and multiplying the resulting integral by $\mathrm{e}^{\mathrm{i} \omega g(\xi)}$. If the integral has multiple stationary points, we can write it as multiple integrals of this form.

The method used in the previous section does not work as the stationary point becomes a singularity when we attempt to integrate by parts. Fortunately, it is possible to remove the singularity (for simplicity we assume that $r=2$ ):

$$
\begin{aligned}
I[f]= & I[f-f(\xi)]+f(\xi) I[1] \\
= & \frac{1}{\mathrm{i} \omega} \int_{a}^{b} \frac{f(x)-f(\xi)}{g^{\prime}(x)} \frac{\mathrm{d}}{\mathrm{d} x} \mathrm{e}^{\mathrm{i} \omega g(x)} \mathrm{d} x+f(\xi) I[1] \\
= & \frac{1}{\mathrm{i} \omega}\left[\frac{f(b)-f(\xi)}{g^{\prime}(b)} \mathrm{e}^{\mathrm{i} \omega g(b)}-\frac{f(a)-f(\xi)}{g^{\prime}(a)} \mathrm{e}^{\mathrm{i} \omega g(a)}\right] \\
& \quad-\frac{1}{\mathrm{i} \omega} I\left[\frac{\mathrm{d}}{\mathrm{d} x}\left[\frac{f(x)-f(\xi)}{g^{\prime}(x)}\right]\right]+f(\xi) I[1] .
\end{aligned}
$$

Again the error term $I\left[\frac{\mathrm{d}}{\mathrm{d} x}\left[\frac{f(x)-f(\xi)}{g^{\prime}(x)}\right]\right]$ is an oscillatory integral, and 
hence we can use induction to obtain a full asymptotic expansion. If there are higher order stationary points, we can subtract out a polynomial to ensure both the function value and necessary derivatives of the integrand vanish in order to make the singularity removable. We thus obtain the following theorem. To simplify subsequent developments, we introduce the Levin differential operator $\mathcal{L}[v]=v^{\prime}+\mathrm{i} \omega g^{\prime} v$, which has the property $I[\mathcal{L}[v]]=v(b) \mathrm{e}^{\mathrm{i} \omega g(b)}-v(a) \mathrm{e}^{\mathrm{i} \omega g(a)}$, as explained in Section 1.4:

Theorem 2 [19] Define $\mu[f]=\sum_{k=0}^{r-2} \frac{f^{k}(\xi)}{k !} \mu_{k}(x)$, where $\mathcal{L}\left[\mu_{k}\right](x)=x^{k}$ for $\mathcal{L}[v]=v^{\prime}+\mathrm{i} \omega g^{\prime} v$. Furthermore, let

$$
\sigma_{0}(x)=f(x), \quad \sigma_{k+1}(x)=\frac{\mathrm{d}}{\mathrm{d} x} \frac{\sigma_{k}(x)-\mathcal{L}\left[\mu\left[\sigma_{k}\right]\right](x)}{g^{\prime}(x)} .
$$

Then

$$
\begin{gathered}
I[f] \sim \sum_{k=0}^{\infty} \frac{1}{(-\mathrm{i} \omega)^{k}}\left\{\mu\left[\sigma_{k}\right](b) \mathrm{e}^{\mathrm{i} \omega g(b)}-\mu\left[\sigma_{k}\right](a) \mathrm{e}^{\mathrm{i} \omega g(a)}\right\} \\
-\sum_{k=0}^{\infty} \frac{1}{(-\mathrm{i} \omega)^{k+1}}\left\{\frac{\sigma_{k}(b)-\mathcal{L}\left[\mu\left[\sigma_{k}\right]\right](b)}{g^{\prime}(b)} \mathrm{e}^{\mathrm{i} \omega g(b)}\right. \\
\left.-\frac{\sigma_{k}(a)-\mathcal{L}\left[\mu\left[\sigma_{k}\right]\right](a)}{g^{\prime}(a)} \mathrm{e}^{\mathrm{i} \omega g(a)}\right\} .
\end{gathered}
$$

This asymptotic expansion depends on knowledge of the moments

$$
I\left[x^{k}\right]=I\left[\mathcal{L}\left[\mu_{k}\right]\right]=\mu_{k}(b) \mathrm{e}^{\mathrm{i} \omega g(b)}-\mu_{k}(a) \mathrm{e}^{\mathrm{i} \omega g(a)},
$$

which is less than ideal. The required functions $\mu_{k}$ are known in closed form only when $g(x)=x^{r}$. But we need not use the polynomials $x^{k}$ in the preceding construction: any basis that can interpolate $f$ and its derivatives at the stationary point will do. Thus we can construct a basis so that the moments are known in closed form for any $g$. A good choice was presented in [30]. Define

$$
\begin{aligned}
\phi_{r, k}(x)=D_{r, k}(\operatorname{sgn}(x-\xi)) & \frac{\omega^{-\frac{k+1}{r}}}{r} \mathrm{e}^{-\mathrm{i} \omega g(x)+\frac{1+k}{2 r} \mathrm{i} \pi} \\
& \times\left[\Gamma\left(\frac{1+k}{r},-\mathrm{i} \omega g(x)\right)-\Gamma\left(\frac{1+k}{r}, 0\right)\right],
\end{aligned}
$$

where

$$
D_{r, k}(s)= \begin{cases}(-1)^{k} & s<0 \text { and } r \text { even } \\ (-1)^{k} \mathrm{e}^{-\frac{1+k}{r} \mathrm{i} \pi} & s<0 \text { and } r \text { odd } \\ -1 & \text { otherwise. }\end{cases}
$$


Then

$$
\mathcal{L}\left[\phi_{r, k}\right](x)=\operatorname{sgn}(x)^{r+k+1} \frac{|g(x)|^{\frac{k+1}{r}-1} g^{\prime}(x)}{r},
$$

which is a $C^{\infty}$ Chebyshev set, hence can interpolate any function at a sequence of nodes and multiplicities. Furthermore, we can compute the moments with respect to this basis:

$$
I\left[\mathcal{L}\left[\phi_{r, k}\right]\right]=\phi_{r, k}(b) \mathrm{e}^{\mathrm{i} \omega g(b)}-\phi_{r, k}(a) \mathrm{e}^{\mathrm{i} \omega g(a)} .
$$

We obtain an equivalent theorem to Theorem 2 using this basis:

Theorem 3 [30] Define $\mu[f]=\sum_{k=0}^{r-2} c_{k} \phi_{r, k}$ so that

$$
\mathcal{L}[\mu[f]](\xi)=f(\xi), \ldots, \mathcal{L}[\mu[f]]^{(r-2)}(\xi)=f^{(r-2)}(\xi) .
$$

Furthermore, let

$$
\sigma_{0}(x)=f(x), \quad \sigma_{k+1}(x)=\frac{\mathrm{d}}{\mathrm{d} x} \frac{\sigma_{k}(x)-\mathcal{L}\left[\mu\left[\sigma_{k}\right]\right](x)}{g^{\prime}(x)} .
$$

Then

$$
\begin{gathered}
I[f] \sim \sum_{k=0}^{\infty} \frac{1}{(-\mathrm{i} \omega)^{k}}\left\{\mu\left[\sigma_{k}\right](b) \mathrm{e}^{\mathrm{i} \omega g(b)}-\mu\left[\sigma_{k}\right](a) \mathrm{e}^{\mathrm{i} \omega g(a)}\right\} \\
-\sum_{k=0}^{\infty} \frac{1}{(-\mathrm{i} \omega)^{k+1}}\left\{\frac{\sigma_{k}(b)-\mathcal{L}\left[\mu\left[\sigma_{k}\right]\right](b)}{g^{\prime}(b)} \mathrm{e}^{\mathrm{i} \omega g(b)}\right. \\
\left.-\frac{\sigma_{k}(a)-\mathcal{L}\left[\mu\left[\sigma_{k}\right]\right](a)}{g^{\prime}(a)} \mathrm{e}^{\mathrm{i} \omega g(a)}\right\} .
\end{gathered}
$$

The first term in this expansion is equivalent to the method of stationary phase [29], though derived in a different manner.

\subsubsection{Multivariate asymptotic expansion}

A multivariate asymptotic expansion can be derived in a very similar manner to the univariate asymptotic expansion, where a partial integration formula based on generalized Stokes' theorem is used. The expansion no longer has a simple explicit form, but the key point of the theorem is that the asymptotics depends on the vertices of the domain. There is an additional criteria known as the nonresonance condition, which is satisfied whenever $\nabla g$ does not vanish and is not orthogonal to the boundary. This is similar to requiring the absence of stationary points in one dimension. 
Theorem 4 [21] Suppose that the nonresonance condition is satisfied. Then, for $\omega \rightarrow \infty$,

$$
I_{g}[f, \Omega] \sim \sum_{k=0}^{\infty} \frac{1}{(-\mathrm{i} \omega)^{k+d}} \Theta_{k}[f],
$$

where $\Theta_{k}[f]$ depends on $f^{(\boldsymbol{m})}$ for $|\boldsymbol{m}| \leq k$, where $|\boldsymbol{m}|$ is the sum of the components of $\boldsymbol{m}$, evaluated at the vertices of $\Omega$.

\subsection{Filon method}

Though the importance of asymptotic methods cannot be overstated, the lack of convergence forces us to look for alternative numerical schemes. In practice the frequency of oscillations is fixed, and the fact that an approximation method is more accurate for higher frequency is irrelevant; all that matters is that the error for the given integral is small. Thus, though asymptotic expansions lie at the heart of oscillatory quadrature, they are not useful in and of themselves unless the frequency is extremely large. In a nutshell, the basic goal of oscillatory quadrature, then, is to find and investigate methods which preserve the asymptotic properties of an asymptotic expansion, whilst allowing for arbitrarily high accuracy for a fixed frequency. Having been spoilt by the pleasures of asymptotic expansions, we also want methods such that the order of operations is independent of $\omega$, and comparable in cost to the evaluation of the expansion. Fortunately, methods have been developed with these properties, in particular the Filon method and Levin collocation method.

The first known numerical quadrature scheme for oscillatory integrals was developed in 1928 by Louis Napoleon George Filon [11]. Filon presented a method for efficiently computing the Fourier integral

$$
\int_{a}^{b} f(x) \sin \omega x \mathrm{~d} x .
$$

As originally constructed, the method consists of dividing the interval into $2 n$ panels of size $h$, and applying a modified Simpson's rule on each panel. In other words, $f$ is interpolated at the endpoints and midpoint of each panel by a quadratic. In each panel the integral becomes a polynomial multiplied by the oscillatory kernel $\sin \omega x$, which can be integrated in closed form. This method was generalized in [27] by using higher degree polynomials in each panel, again with evenly spaced nodes. We refer to any method where $f$ is approximated by a polynomial $v=$ 
$\sum c_{k} x^{k}$ which is integrated exactly as a Filon-type method. Thus we define

$$
Q^{F}[f]=I[v]=\sum c_{k} I\left[x^{k}\right] .
$$

We also can obtain a bound via the Cauchy-Shwarz inequality which shows that a Filon-type method converges whenever the polynomial approximation converges in the $L_{2}$ norm:

$$
\left|I[f]-Q^{F}[f]\right|=|I[f-v]| \leq \sqrt{b-a}\|f-v\|_{2} .
$$

\subsubsection{Univariate Filon-type methods}

Theorem 1 has an interesting consequence for oscillatory integrals without stationary points: if $f$ and its first $s-1$ derivatives vanish at the endpoints of the interval, then the oscillatory integral itself decays like $\mathcal{O}\left(\omega^{-s-1}\right)$. The error of a Filon-type method is itself a highly oscillatory integral. Thus if $v$ interpolates the first $s-1$ derivatives of $f$ at the endpoints we know immediately from the asymptotic expansion that the error decays like

$$
I[f]-Q^{F}[f]=I[f-v] \sim \mathcal{O}\left(\omega^{-s-1}\right) .
$$

Thus we can use Hermite interpolation to achieve higher asymptotic orders. Choose a sequence of nodes $\left\{x_{1}, \ldots, x_{\nu}\right\}$, where $x_{1}=a$ and $x_{\nu}=b$, and associate a sequence of multiplicities $m_{k}$. We then determine $v=\sum c_{k} x^{k}$ by solving the following system:

$$
v\left(x_{k}\right)=f\left(x_{k}\right), \ldots, v^{\left(m_{k}-1\right)}\left(x_{k}\right)=f^{\left(m_{k}-1\right)}\left(x_{k}\right), \quad k=1,2, \ldots, \nu .
$$

In this case, $s=\min \left\{m_{1}, m_{\nu}\right\}$. This method was proposed in [19]. Without delving into details, we note that evaluation of derivatives can be replaced by function evaluation at points that coalesce at the vertices as $\omega$ increases [18].

If the integral has stationary points, interpolation at the endpoints is not sufficient to accelerate the asymptotic decay. This follows since the asymptotic expansion now depends on $f$ at the stationary point as well. In order to achieve a decay rate of $\mathcal{O}\left(\omega^{-s-1 / r}\right)$ we thus need to interpolate $f$ and its first $r s-2$ derivatives at the stationary point [30], which follows from Theorem 2, L'Hôpital's rule, and the fact that the integral itself decays like $\mathcal{O}\left(\omega^{-1 / r}\right)$ [35].

As a simple example, consider the integral

$$
I[f]=\int_{0}^{1} \frac{x+3}{x+1} \mathrm{e}^{\mathrm{i} \omega x} \mathrm{~d} x .
$$




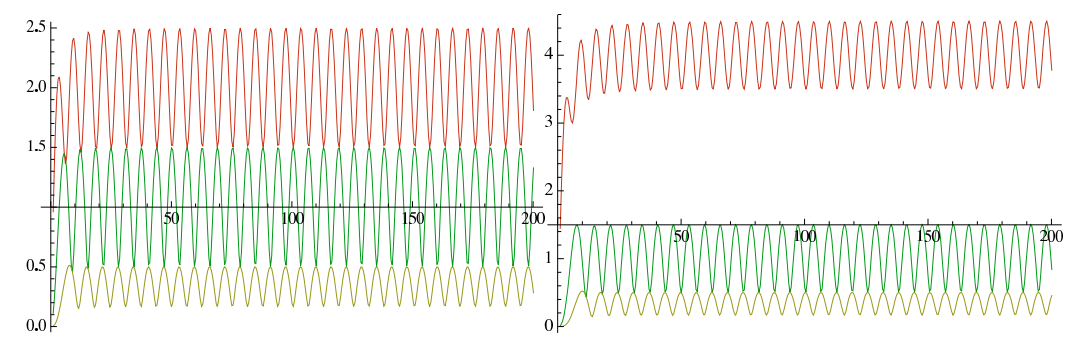

Fig. 1.3. The error in approximating $\int_{0}^{1} \frac{x+3}{x+1} \mathrm{e}^{\mathrm{i} \omega x} \mathrm{~d} x$. In the left graph, we scale the error by $\omega^{2}$ and compare the one-term asymptotic expansion (top) and Filon-type methods with nodes $\{0,1\}$ and multiplicities both one (middle) and nodes $\left\{0, \frac{1}{2}, 1\right\}$ and multiplicities all one (bottom). In the right graph, we scale the error by $\omega^{3}$ and we compare the two-term asymptotic expansion (top) and Filon-type methods with nodes $\{0,1\}$ and multiplicities both two (middle) and nodes $\left\{0, \frac{1}{2}, 1\right\}$ and multiplicities $\{2,1,2\}$ (bottom).

In Figure 1.3, we compare the asymptotic expansion to four Filon-type methods: two of order $\mathcal{O}\left(\omega^{-2}\right)$ and two of order $\mathcal{O}\left(\omega^{-3}\right)$. As can be seen, Filon-type methods allow us to considerably decrease the error compared to the asymptotic expansion, even when only a few nodes are used.

\subsubsection{Moment-free Filon-type methods}

The computation of the Filon approximation rests on the ability to compute the moments

$$
\int_{a}^{b} x^{k} \mathrm{e}^{\mathrm{i} \omega x} \mathrm{~d} x
$$

For this particular oscillator the moments are computable in closed form, either through integration by parts or by the identity

$$
\int_{a}^{b} x^{k} \mathrm{e}^{\mathrm{i} \omega x} \mathrm{~d} x=\frac{1}{(-\mathrm{i} \omega)^{k+1}}[\Gamma(1+k,-\mathrm{i} \omega a)-\Gamma(1+k,-\mathrm{i} \omega b)],
$$

where $\Gamma$ is the incomplete Gamma function [1]. But often in applications we have irregular oscillators, giving us integrals of the form

$$
\int_{a}^{b} f(x) \mathrm{e}^{\mathrm{i} \omega g(x)} \mathrm{d} x .
$$

In this case knowledge of moments depends on the oscillator $g$. If we are fortunate, the moments are still known, and Filon-type methods are 
applicable. This is true if $g$ is a polynomial of degree at most two or if $g(x)=x^{r}$. But we need not step too far outside the realm of these simple examples before explicit moment calculation falls apart: moments are not even known for $g(x)=x^{3}-x$ nor $g(x)=\cos x$. Even when moments are known, they are typically known in terms of special functions, such as the incomplete Gamma function or more generally the hypergeometric function [1]. The former of these is efficiently computable [36]. The latter, on the other hand, are significantly harder to compute for the invariably large parameters needed, though some computational schemes exist $[12,28,26]$. Thus it is necessary that we find an alternative to the Filon method.

We are not required to use polynomial interpolation, however, and results from Subsection 1.2.2 present an intriguing alternative proposed in [30]: use the basis

$$
\mathcal{L}\left[\phi_{r, k}\right]=\operatorname{sgn}(x)^{r+k+1} \frac{|g(x)|^{\frac{k+1}{r}-1} g^{\prime}(x)}{r}
$$

in place of the polynomial basis $x^{k}$. We know this basis has two important properties: its moments are computable and it forms a Chebyshev set. Thus given a sequence of nodes $\left\{x_{1}, \ldots, x_{\nu}\right\}$ and multiplicities $\left\{m_{1}, \ldots, m_{\nu}\right\}$ we can successfully solve the system

$$
\mathcal{L}[v]\left(x_{k}\right)=f\left(x_{k}\right), \ldots, \mathcal{L}[v]^{\left(m_{k}-1\right)}\left(x_{k}\right)=f^{\left(m_{k}-1\right)}\left(x_{k}\right), \quad k=1, \ldots, \nu,
$$

for the unknowns $c_{k}$, where $v=\sum c_{k} \phi_{r, k}$. As in the standard Filontype method, the asymptotic order depends on the number of derivatives interpolated at the endpoints and stationary point.

We now approximate the integral

$$
I[f]=\int_{0}^{1} \frac{x+3}{x+2} \mathrm{e}^{\mathrm{i} \omega(1-\cos x)} \mathrm{d} x .
$$

In Figure 1.4, we compare the moment-free asymptotic expansion to four Moment-free Filon-type methods: two of order $\mathcal{O}\left(\omega^{-3 / 2}\right)$ and two of order $\mathcal{O}\left(\omega^{-5 / 2}\right)$. Again, we can reduce the error of Filon-type methods by adding interior interpolation points.

\subsubsection{Multivariate Filon-type methods}

We can readily derive a Filon-type method for multivariate oscillatory integrals as well [21]. As before, we interpolate a multivariate function $f$ 


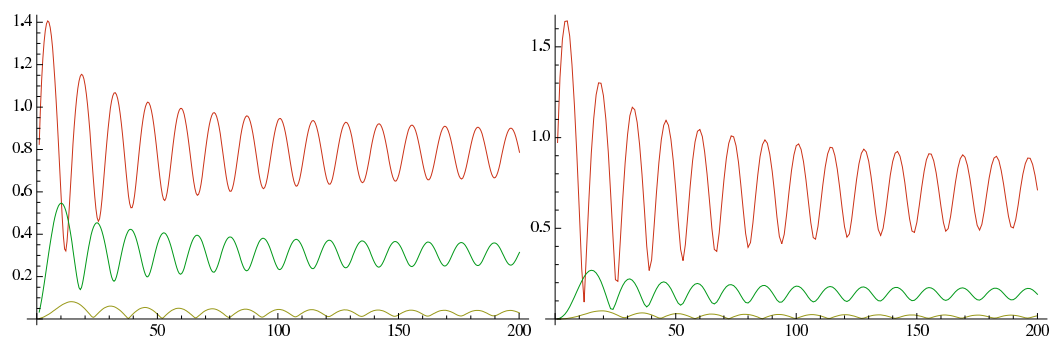

Fig. 1.4. The error in approximating $\int_{-1}^{1} \frac{x+3}{x+2} \mathrm{e}^{\mathrm{i} \omega(1-\cos x)} \mathrm{d} x$. In the left graph, we scale the error by $\omega^{3 / 2}$ and compare the one-term moment-free asymptotic expansion (top) and Moment-free Filon-type methods with nodes $\{-1,0,1\}$ and multiplicities all one (middle) and nodes $\left\{-1,-\frac{1}{2}, 0, \frac{1}{2}, 1\right\}$ and multiplicities all one (bottom). In the right graph, we scale the error by $\omega^{5 / 2}$ and we compare the two-term moment-free asymptotic expansion (top) and Momentfree Filon-type methods with nodes $\{-1,0,1\}$ and multiplicities $\{2,3,2\}$ (middle) and nodes $\left\{-1,-\frac{1}{2}, 0, \frac{1}{2}, 1\right\}$ and multiplicities $\{2,1,3,1,2\}$ (bottom).

by a polynomial $v$ at a sequence of points $\left\{\boldsymbol{x}_{1}, \ldots, \boldsymbol{x}_{\nu}\right\}$, again with multiplicities $\left\{m_{1}, \ldots, m_{\nu}\right\}$. Assuming such an interpolation is possiblewhich, unlike univariate polynomial interpolation, is not ensured - we obtain the approximation:

$$
Q^{F}[f]=I[v]=\int_{\Omega} v(\boldsymbol{x}) \mathrm{e}^{\mathrm{i} \omega g(\boldsymbol{x})} \mathrm{d} V .
$$

Again, this requires knowledge of the moments $I\left[x_{1}^{i_{1}} \ldots x_{d}^{i_{2}}\right]$, whose evaluation depends not only on the oscillator $g$ but also the domain $\Omega$. There are few cases where these moments are known, but they can be found in closed form if $\Omega$ is a simplex and $g(\boldsymbol{x})=\boldsymbol{\kappa} \cdot \boldsymbol{x}$ is linear. If $g$ is linear, a composite Filon-type rule can be constructed by triangulating $\Omega$, and applying a Filon-type method to each triangle in the mesh.

The asymptotic order of a multivariate Filon-type method follows immediately from Theorem 4, as long as $\nabla g=\boldsymbol{\kappa}$ is not orthogonal to any of the faces of the boundary. Thus if we interpolate at the vertices with multiplicities at least $s$, we achieve an order

$$
Q^{F}[f]-I[f] \sim \mathcal{O}\left(\omega^{-s-d}\right) .
$$

Let $S=\{(x, y): x \geq 0, y \geq 0$ and $x+y \leq 1\}$ be the two-dimensional simplex. We now approximate the integral

$$
\iint_{S} \frac{\mathrm{e}^{\mathrm{i} \omega(x-y)}}{2 x+y+1} \mathrm{~d} V=\int_{0}^{1} \int_{0}^{1-x} \frac{\mathrm{e}^{\mathrm{i} \omega(x-y)}}{2 x+y+1} \mathrm{~d} y \mathrm{~d} x .
$$




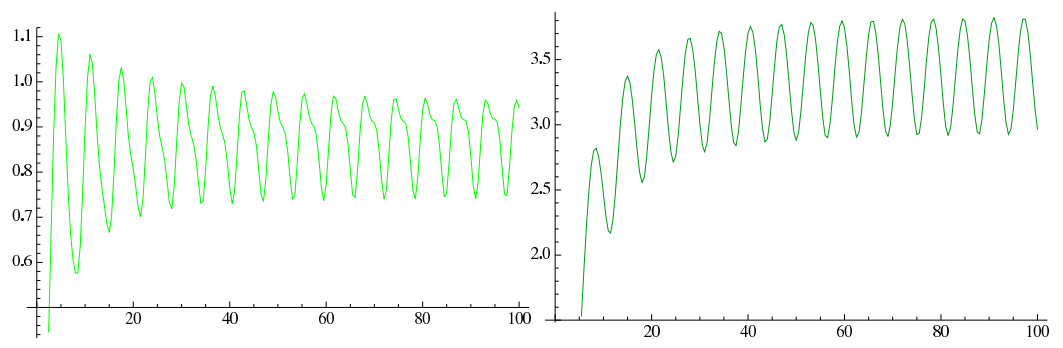

Fig. 1.5. The error of two Filon-type methods in approximating $\iint_{S} \frac{\mathrm{e}^{\mathrm{i} \omega(x-y)}}{2 x+y+1} \mathrm{~d} V$. In the left graph, we scale the error by $\omega^{3}$ where we interpolate at the vertices with multiplicities all one. In the right graph, we scale by $\omega^{4}$ for interpolating at $\left\{(0,0),(1,0),(0,1),\left(\frac{1}{3}, \frac{1}{3}\right)\right\}$ with multiplicities $\{2,2,2,1\}$.

Simply by interpolating at the vertices of the domain, we obtain an asymptotic order $\mathcal{O}\left(\omega^{-3}\right)$, as seen in the left graph of Figure 1.5. The right graph demonstrates that increasing multiplicities at the vertices does indeed increase the asymptotic order to $\mathcal{O}\left(\omega^{-4}\right)$.

\subsection{Levin collocation method}

In 1982, David Levin developed the Levin collocation method [24], which approximates oscillatory integrals free of stationary points without using moments. A function $F$ such that $\frac{\mathrm{d}}{\mathrm{d} x}\left[F \mathrm{e}^{\mathrm{i} \omega g}\right]=f \mathrm{e}^{\mathrm{i} \omega g}$ satisfies

$$
I[f]=\int_{a}^{b} f \mathrm{e}^{\mathrm{i} \omega g} \mathrm{~d} x=\int_{a}^{b} \frac{\mathrm{d}}{\mathrm{d} x}\left[F \mathrm{e}^{\mathrm{i} \omega g}\right] \mathrm{d} x=F(b) \mathrm{e}^{\mathrm{i} \omega g(b)}-F(a) \mathrm{e}^{\mathrm{i} \omega g(a)} .
$$

By expanding out the derivatives, we can rewrite this condition as $\mathcal{L}[F]=f$ for the operator

$$
\mathcal{L}[F]=F^{\prime}+\mathrm{i} \omega g^{\prime} F
$$

If we can approximate the function $F$, then we can approximate $I[f]$ easily. In order to do so, we use collocation with the operator $\mathcal{L}$. Let $v=\sum_{k=1}^{\nu} c_{k} \psi_{k}$ for some basis $\left\{\psi_{1}, \ldots, \psi_{\nu}\right\}$. Given a sequence of collocation nodes $\left\{x_{1}, \ldots, x_{\nu}\right\}$, we determine the coefficents $c_{k}$ by solving the collocation system

$$
\mathcal{L}[v]\left(x_{1}\right)=f\left(x_{1}\right), \ldots, \mathcal{L}[v]\left(x_{\nu}\right)=f\left(x_{\nu}\right) .
$$


We can then define the approximation $Q^{L}[f]$ to be

$Q^{L}[f]=\int_{a}^{b} \mathcal{L}[v] \mathrm{e}^{\mathrm{i} \omega g} \mathrm{~d} x=\int_{a}^{b} \frac{\mathrm{d}}{\mathrm{d} x}\left[v \mathrm{e}^{\mathrm{i} \omega g}\right] \mathrm{d} x=v(b) \mathrm{e}^{\mathrm{i} \omega g(b)}-v(a) \mathrm{e}^{\mathrm{i} \omega g(a)}$.

Levin was the first to note the asymptotic properties of oscillatory quadrature schemes, as well as the importance of endpoints in the collocation system. This method has an error $I[f]-Q^{L}[f]=\mathcal{O}\left(\omega^{-1}\right)$ when the endpoints of the interval are not included in the collocation nodes. When the endpoints are included, on the other hand, the asymptotic order increases to $I[f]-Q^{L}[f]=\mathcal{O}\left(\omega^{-2}\right)$.

A Levin collocation method was also constructed for oscillatory integrals over a square. In this case a Levin differential operator was constructed by iterating the method for each dimension. Though we do investigate multivariate Levin-type methods in Subsection 1.4.3, we will not use this construction as it is limited to hypercubes.

\subsubsection{Univariate Levin-type methods}

Motivated by the asymptotic expansion and the results for Filon-type methods, we can now develop Levin-type methods, as proposed in [31]. In addition to collocating function values, we also collocate derivatives at the collocation points $\left\{x_{1}, \ldots, x_{\nu}\right\}$, up to given multiplicities $\left\{m_{1}, \ldots, m_{\nu}\right\}$. In other words, for $v=\sum c_{k} \psi_{k}$, we determine the unknown coefficients by solving the system

$$
\mathcal{L}[v]\left(x_{k}\right)=f\left(x_{k}\right), \ldots, \mathcal{L}[v]^{\left(m_{k}-1\right)}\left(x_{k}\right)=f^{\left(m_{k}-1\right)}\left(x_{k}\right), \quad k=1, \ldots, \nu .
$$

If the basis $\left\{\psi_{1}, \psi_{2}, \ldots\right\}$ itself can interpolate at the points and multiplicities, then large $\omega$ ensures a solution to this system exists, and each of the coefficients is $\mathcal{O}\left(\omega^{-1}\right)$. This can be seen via the following logic: we can write (1.3) as $(P+\mathrm{i} \omega G) \boldsymbol{c}=\boldsymbol{f}$, where $G$ is the Vandermonde-like matrix obtained from evaluating $g^{\prime} \psi_{k}$ at the nodes and multiplicities. Thus $G$ is nonsingular, and

$$
(P+\mathrm{i} \omega G)^{-1}=G^{-1}(\mathrm{i} \omega)^{-1}(I+o(1))^{-1} .
$$

It is clear that $(I+o(1))^{-1} \rightarrow I$, thus this expression is $\mathcal{O}\left(\omega^{-1}\right)$, and hence $\boldsymbol{c}=(P+\mathrm{i} \omega G)^{-1} \boldsymbol{f}=\mathcal{O}\left(\omega^{-1}\right)$. It follows that $\mathcal{L}[v]$ and its derivatives are all bounded as $\omega \rightarrow \infty$, thus again we can utilize the asymptotic expansion to show that

$$
I[f]-Q^{L}[f]=I[f-\mathcal{L}[v]] \sim \mathcal{O}\left(\omega^{-s-1}\right),
$$




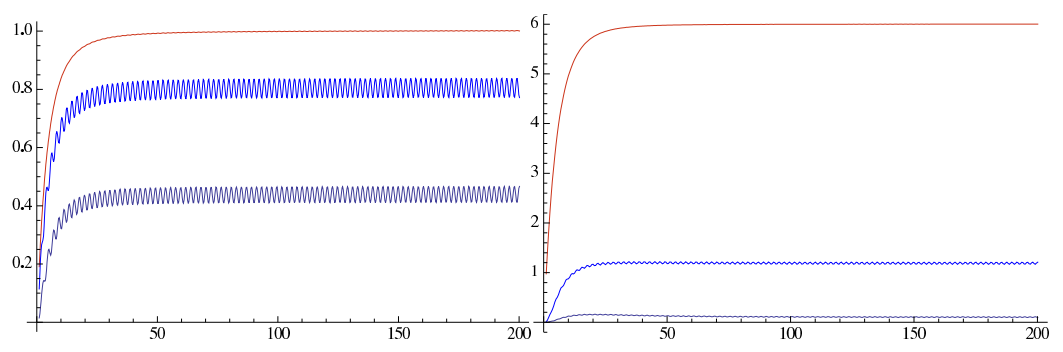

Fig. 1.6. The error in approximating $\int_{0}^{1} \sinh x \mathrm{e}^{\mathrm{i} \omega\left(x^{3}+x^{2}+x\right)} \mathrm{d} x$. In the left graph, we scale the error by $\omega^{2}$ and compare the one-term asymptotic expansion (top) and Levin-type methods with nodes $\{0,1\}$ and multiplicities both one (middle) and nodes $\left\{0, \frac{1}{2}, 1\right\}$ and multiplicities all one (bottom). In the right graph, we scale the error by $\omega^{3}$ and we compare the two-term asymptotic expansion (top) and Levin-type methods with nodes $\{0,1\}$ and multiplicities both two (middle) and nodes $\left\{0, \frac{1}{2}, 1\right\}$ and multiplicities $\{2,1,2\}$ (bottom).

for $s=\min \left\{m_{1}, m_{\nu}\right\}$.

An example of an integral which cannot be computed via Filon-type methods is

$$
I[f]=\int_{0}^{1} \sinh x \mathrm{e}^{\mathrm{i} \omega\left(x^{3}+x^{2}+x\right)} \mathrm{d} x .
$$

We compare the asymptotic expansion to two Levin-type methods in Figure 1.6. We use the same nodes and multiplicities as in the Filontype methods of Figure 1.3, and see the same increase of asymptotic order with the use of multiplicities.

\subsubsection{Chung, Evans E Webster methods}

Often methods represented as weighted sums are preferred. Though Filon and Levin collocation methods are extremely powerful, they do not fall into this framework. In [9], Evans and Webster construct such a method for irregular exponential oscillators, based on the Levin collocation method. We want to choose weights $w_{k}$ and nodes $x_{k}$ such that

$$
\int_{-1}^{1} \phi_{k}(x) \mathrm{e}^{\mathrm{i} \omega g(x)} \mathrm{d} x=\sum_{k=0}^{n} w_{k} \phi_{k}\left(x_{k}\right)
$$

for some suitable basis $\phi_{k}$. Unlike Gaussian quadrature, we do not choose $\phi_{k}$ to be polynomials. Instead, we choose them based on the 
Levin differential equation:

$$
\phi_{k}=\mathcal{L}\left[T_{k}\right]=T_{k}^{\prime}+\mathrm{i} \omega g^{\prime} T_{k},
$$

where $T_{k}$ are the Chebyshev polynomials. The moments with respect to $\phi_{k}$ are computable in closed form:

$$
\int_{-1}^{1} \phi_{k}(x) \mathrm{e}^{\mathrm{i} \omega g(x)} \mathrm{d} x=T_{k}(1) \mathrm{e}^{\mathrm{i} \omega g(1)}-T_{k}(-1) \mathrm{e}^{\mathrm{i} \omega g(-1)} .
$$

We can thus determine suitable weights and nodes. Numerical results suggest that this preserves the asymptotic niceties of the original Levin collocation method.

\subsubsection{Multivariate Levin-type methods}

We can generalize Levin-type methods to multivariate domains [32]. The construction of the collocation operator $\mathcal{L}$ followed from the fundamental theorem of calculus, which allowed us to express the value of the integral in terms of a function evaluated at the endpoints of the interval. The construction of a multivariate version will proceed in the same manner, where we use generalized Stokes' theorem to express the value of the integral as an integral over the boundary of the domain.

Define

$$
\mathcal{L}[\boldsymbol{v}]=\nabla \cdot \boldsymbol{v}+\mathrm{i} \omega \nabla g \cdot \boldsymbol{v} .
$$

Generalized Stokes' theorem informs us that

$$
\int_{\partial \Omega} \mathrm{e}^{\mathrm{i} \omega g} \boldsymbol{v} \cdot \mathrm{d} \boldsymbol{s}=\int_{\Omega} \nabla \cdot\left(\mathrm{e}^{\mathrm{i} \omega g} \boldsymbol{v}\right) \mathrm{d} V=\int_{\Omega} \mathcal{L}[\boldsymbol{v}] \mathrm{e}^{\mathrm{i} \omega g} \mathrm{~d} V=I[\mathcal{L}[\boldsymbol{v}]],
$$

where $\mathrm{d} \boldsymbol{s}=(\mathrm{d} y,-\mathrm{d} x)^{\top}$. Thus we use collocation at $\left\{\boldsymbol{x}_{1}, \ldots, \boldsymbol{x}_{\nu}\right\}$ with multiplicities $\left\{m_{1}, \ldots, m_{\nu}\right\}$ to determine the coefficients of $\boldsymbol{v}=\sum c_{k} \boldsymbol{\psi}_{k}$, i.e., we solve the system

$$
\mathcal{L}[\boldsymbol{v}]^{(\boldsymbol{m})}\left(\boldsymbol{x}_{k}\right)=f^{(\boldsymbol{m})}\left(\boldsymbol{x}_{k}\right), \quad \text { for } \quad|\boldsymbol{m}|<m_{k}, \quad k=1, \ldots, \nu,
$$

where $|\boldsymbol{m}|$ expresses the sum of the individual components. Similar to the univariate case, this system is guaranteed to have a solution for large $\omega$ whenever the basis $\nabla g \cdot \boldsymbol{\psi}_{k}$ can interpolate at the nodes and multiplicities. Hence we obtain

$$
I[f] \approx I[\mathcal{L}[\boldsymbol{v}]]=\int_{\partial \Omega} \mathrm{e}^{\mathrm{i} \omega g} \boldsymbol{v} \cdot \mathrm{d} \boldsymbol{s} .
$$

We have approximated the oscillatory integral by another oscillatory 

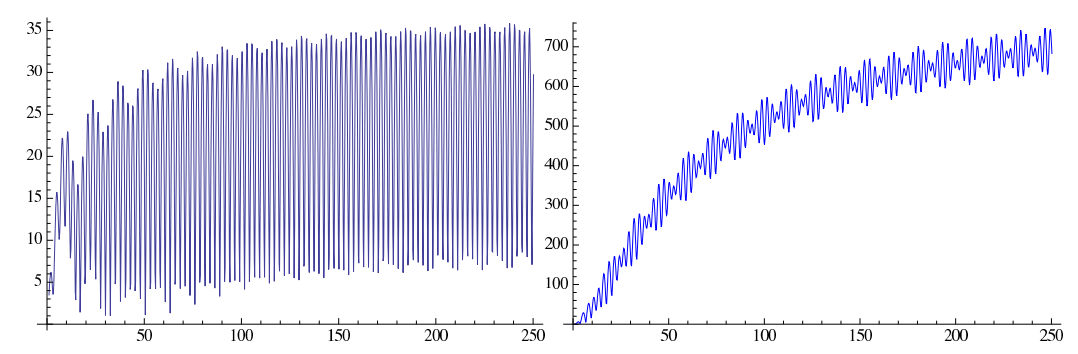

Fig. 1.7. The error of two Levin-type methods in approximating $\iint_{H}\left(\mathrm{e}^{x y}+x^{2}+\right.$ 1) $\mathrm{e}^{\mathrm{i} \omega\left(\cos \left(\frac{x}{2}+1\right)+y^{2}+y\right)} \mathrm{d} V$. In the left graph, we scale the error by $\omega^{3}$ where we interpolate at the vertices with multiplicities all one. In the right graph, we scale by $\omega^{4}$ for interpolating at $\left\{(0,0),(1,0),(0,1),\left(\frac{1}{4}, \frac{1}{4}\right)\right\}$ with multiplicities $\{2,2,2,1\}$.

integral of one dimension less. We can thus iterate the procedure on each dimension, eventually arriving at univariate integrals, which we know how to approximate. It is possible to prove that like multivariate Filon-type methods, an error of order $\mathcal{O}\left(\omega^{-s-d}\right)$ is achieved when the function value and derivatives of order $s-1$ of $f$ are collocated at each vertex. This does require that $s-1$ derivatives are used for each of the vertices of the boundary integrals as well.

In our example, we define $\boldsymbol{\psi}_{k}$ as a constant vector $(1,-1)^{\top}$ times the standard multivariate polynomial basis. Thus each $\psi_{k}$ is of the form $(1,-1)^{\top} x^{i} y^{j}$. Letting $H=\left\{(x, y): x \geq 0, y \geq 0\right.$ and $\left.x^{2}+y^{2} \leq 1\right\}$ denote a quarter-disc, in Figure 1.7 we approximate the integral

$$
\begin{aligned}
\iint_{H}\left(\mathrm{e}^{x y}+x^{2}+1\right) \mathrm{e}^{\mathrm{i} \omega\left(\cos \left(\frac{x}{2}+1\right)+y^{2}+y\right)} \mathrm{d} V \\
\quad=\int_{0}^{1} \int_{0}^{\sqrt{1-x^{2}}}\left(\mathrm{e}^{x y}+x^{2}+1\right) \mathrm{e}^{\mathrm{i} \omega\left(\cos \left(\frac{x}{2}+1\right)+y^{2}+y\right)} \mathrm{d} y \mathrm{~d} x .
\end{aligned}
$$

Once we push the integral to the boundary we employ three univariate Levin-type methods. In the left graph of this figure we use the endpoints with multiplicities one for each of the univariate methods, in the right graph we use the endpoints with multiplicities two.

\subsection{Steepest descent methods}

An entirely different approach becomes applicable once we assume that both $f$ and $g$ are analytic functions. Then, by deforming the path of 

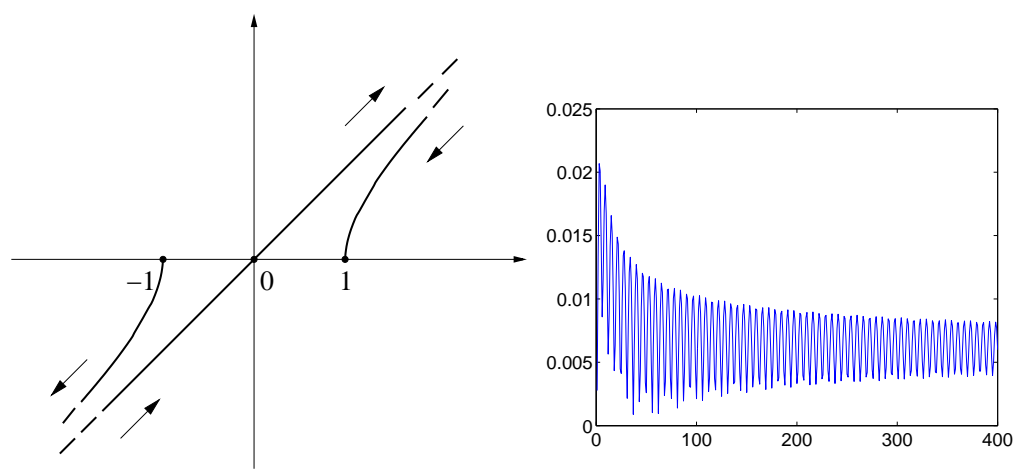

Fig. 1.8. The absolute error in approximating $\int_{-1}^{1} \frac{1}{(x+3)^{2}} \mathrm{e}^{\mathrm{i} \omega x^{2}} \mathrm{~d} x$ as a function of $\omega$ by a numerical steepest descent scheme using 4 quadrature points, 1 near each endpoint and 2 near the stationary point. The error is scaled by $\omega^{5 / 2}$.

integration into the complex plane, efficient numerical schemes can again be devised for the evaluation of $I[f]$ with low computational cost and high asymptotic accuracy. The methods require no moments, but they require computations in the complex plane.

\subsubsection{The path of steepest descent}

For complex valued functions $g(x)$, we observe that the complex exponential function $\mathrm{e}^{\mathrm{i} \omega g(x)}$ is oscillatory only as a function of the real part of $g$. The so-called path of steepest descent originating at the point $x=a$ is such that the real part of $g$ is kept fixed along the path. Assuming a parameterization $h_{a}(p)$ of the path, $p \in[0, P]$, this is achieved by solving

$$
g\left(h_{a}(p)\right)=g(a)+\mathrm{i} p,
$$

subject to the condition $h_{a}(0)=a$. Along such a path, the integrand is nonoscillatory and exponentially decaying, taking the form

$$
\mathrm{e}^{\mathrm{i} \omega g(a)} \int_{0}^{P} f\left(h_{a}(p)\right) h_{a}^{\prime}(p) \mathrm{e}^{-\omega p} \mathrm{~d} p
$$

At a stationary point $x=\xi$, equation (1.4) has several solutions since the inverse of $g$ is a multivalued function. Two solutions can easily be identified such that deformation onto a collection of steepest descent contours is justified by Cauchy's integral theorem. This is shown in the left panel of Figure 1.8 for the case $g(x)=x^{2}$. 


\subsubsection{Univarate method of steepest descent}

The method of steepest descent is traditionally used to obtain the asymptotic expansion of $I[f][37]$. However, the typical divergence of asymptotic expansions is avoided by evaluating integrals of the form (1.5) numerically. An interesting choice is the use of a Gaussian quadrature rule that incorporates the exponential decay into its weight function [14].

For the endpoints $x=a$ or $x=b$, integral (1.5) is approximated by

$$
\frac{\mathrm{e}^{\mathrm{i} \omega g(x)}}{\omega} \sum_{k=1}^{n} w_{k} f\left(h_{x}\left(\frac{x_{k}}{\omega}\right)\right) h_{x}^{\prime}\left(\frac{x_{k}}{\omega}\right)
$$

where $x_{k}$ and $w_{k}$ are the quadrature points and weights of a classical Gauss-Laguerre rule. It was shown in [14] that, using $n$ evaluations of $f$ near $a$ and near $b$, the error in the approximation behaves as $\mathcal{O}\left(\omega^{-2 n-1}\right)$. The asymptotic order thus obtained is roughly twice that of Filon-type methods or Levin-type methods using $n$ derivatives at each endpoint. This comes at a cost of having to compute $h_{x}(p)$.

At a simple stationary point, it is beneficial to use a Gauss-Hermite rule, with the weight function $\mathrm{e}^{-q^{2}}$. For more degenerate stationary points, the optimal Gaussian quadrature rules are no longer classical [6]. In all cases however, the asymptotic order is approximately twice that of Filon-type methods and Levin-type methods, when using the same number of function evaluations of $f$ or its derivatives.

An example is shown in the right panel of Figure 1.8. Using four function evaluations, the method achieves $\omega^{-5 / 2}$ asymptotic error for the integral $\int_{-1}^{1} \frac{1}{(x+3)^{2}} \mathrm{e}^{\mathrm{i} \omega x^{2}} \mathrm{~d} x$. A Filon-type method would require at least seven function values to achieve the same order.

\subsubsection{Multivariate method of steepest descent}

The univariate method of steepest descent can be extended to multivariate oscillatory integrals by recursion. For example, when $f$ and $g$ depend on two variables $x_{1}$ and $x_{2}$, integration with respect to the variable $x_{1}$ may lead to a steepest descent integral of the form

$$
\mathrm{e}^{\mathrm{i} \omega g\left(a, x_{2}\right)} \int_{0}^{P} f\left(h_{1}\left(p, x_{2}\right), x_{2}\right) \frac{\partial h_{1}}{\partial p}\left(p, x_{2}\right) \mathrm{e}^{-\omega p} \mathrm{~d} p,
$$

which compares to (1.5). The steepest descent path $h_{1}$ now is such that

$$
g\left(h_{1}\left(p, x_{2}\right), x_{2}\right)=g\left(a, x_{2}\right)+\mathrm{i} p .
$$


The path depends on the starting point $x_{1}=a$, but also on the second variable $x_{2}$. The recursive process is started by noting that the line integral above is an oscillatory function in $x_{2}$, with the known oscillator $g\left(a, x_{2}\right)$. Thus, the integral of this function with respect to $x_{2}$ may again be evaluated by deforming onto a path of steepest descent $h_{2}(q)$. One ends up integrating along a manifold of steepest descent, which yields a doubly-infinite, non-oscillatory and exponentially decaying integral

$$
\mathrm{e}^{\mathrm{i} \omega g(a, b)} \int_{0}^{Q} \int_{0}^{P} f\left(h_{1}\left(p, h_{2}(q)\right), h_{2}(q)\right) h_{2}^{\prime}(q) \frac{\partial h_{1}}{\partial p}\left(p, h_{2}(q)\right) e^{-\omega(p+q)} \mathrm{d} p \mathrm{~d} q .
$$

This manifold of steepest descent is not unique, as the lack of symmetry in the expression above indicates. The integrals, as in the univariate case, can be evaluated numerically to high asymptotic order [15].

Much like the result of using generalized Stokes' formula, one can see that each recursive step pushes the integral onto the boundary. The new oscillators that appear in the process may in general have stationary points, which we call resonance points. Such resonance points appear on the boundary of the domain as points where $\nabla g \perp \partial \Omega$. Other contributions come from vertices of $\partial \Omega$ and from critical points where $\nabla g=0$.

The method of steepest descent continues to hold for oscillators $g$ with critical points and resonance points. Integrals with very degenerate oscillators however, for example with a vanishing Hessian, are computable in this manner only subject to certain additional conditions [15].

\subsection{Other methods}

There are assorted other numerical methods developed for approximating oscillatory integrals, typically specializing on particular oscillators. We will not investigate these methods in detail, but they are mentioned here for completeness.

\subsubsection{Fourier oscillator methods}

Many methods exist for the Fourier oscillator, which were reviewed in [10]. They all are based on the fact that moments are computable, and hence are Filon-type methods. The Bakhvalov and Vasil'eva method [4] interpolates $f$ by Legendre polynomials $P_{k}$, and uses the fact that the moments of such polynomials are known explicitly:

$$
\int_{-1}^{1} P_{k}(x) \mathrm{e}^{\mathrm{i} \omega x} \mathrm{~d} x=\mathrm{i}^{k}\left(\frac{2 \pi}{\omega}\right)^{\frac{1}{2}} J_{k+\frac{1}{2}}(\omega)
$$


where $J_{k}$ is a Bessel function [1].

A method based on Clenshaw-Curtis quadrature was also devised, where $f$ is interpolated by Chebyshev polynomials $T_{k}$. We do not have simple formulæ for the resulting moments, so the polynomials $T_{k}$ are then expanded into Legendre polynomials and (1.6) is applied [33, 25]. An alternative from [2] is to express the moments in terms of the hypergeometric function ${ }_{0} F_{1}$ [1]. Special functions can be avoided in both these methods by expanding the Legendre or Chebyshev polynomials into the standard polynomial basis $x^{k}$, whose moments can be found via partial integration [3]. This is not effective for large $k$ due to significant cancellation in the expansions [10].

Though it was not observed in any of these papers, all of these Filontype methods - methods based on interpolating $f$ - have the same asymptotic behaviour as the Filon-type methods developed in Section 1.3. If the endpoints of the interval are included in the interpolation nodes, then error decays like $\mathcal{O}\left(\omega^{-2}\right)$; otherwise the error decays at the same rate as the integral $\mathcal{O}\left(\omega^{-1}\right)$. It means that the number of interpolation points required should actually decrease as the frequency of oscillations increases. Thus at high frequencies we never need to utilize large order polynomials in order to obtain accurate results.

Piessens developed a Gaussian quadrature formula with respect to the weight function $\sin x$ over the interval $[-\pi, \pi]$ [34]. It however relies on considering each period separately, thus still requires a large number of function evaluations to obtain accurate approximations. A similar method based on Gaussian quadrature was developed by Zamfirescu [38], and described in [17] (the original paper is in Romanian). We can rewrite the sine Fourier integral as

$$
\int_{0}^{1} f(x) \sin \omega x \mathrm{~d} x=\int_{0}^{1} f(x)(1+\sin \omega x) \mathrm{d} x-\int_{0}^{1} f(x) \mathrm{d} x .
$$

The second of these integrals is nonoscillatory, so standard quadrature methods can be used to approximate its value. The first integral now has a nonnegative weight function, hence we can approximate it by a weighted sum. Since the moments with respect to the weight function are known, we can successfully compute the quadrature weights needed.

A very effective quadrature scheme is developed in [22] for the standard Fourier oscillator. The paper uses a weighted sum of the value of the functon $f$ and its first derivative at evenly spaced nodes, determining the weights so as to maximize the degree of polynomials integrated exactly. It is noted that, for a method of $N$ points, the error behaves 
like $\mathcal{O}\left(\omega^{-N}\right)$. This is generalized to use higher order derivatives of $f$ in [23], resulting in a significant decrease in error.

\subsubsection{Irregular oscillator methods}

There is also a comparison of methods for irregular oscillators in [10]. In addition to the Levin collocation method and the Chung, Evans and Webster method already discussed, there is method developed by Evans in [7] where the transformation $y=g(x)$ is used to convert the irregular oscillator to a standard Fourier oscillator:

$$
I[f]=\int_{g(a)}^{g(b)} \frac{f\left(g^{-1}(y)\right)}{g^{\prime}\left(g^{-1}(y)\right)} \mathrm{e}^{\mathrm{i} \omega y} \mathrm{~d} y .
$$

Once the integral is in this form, a Filon-type method can be employed, in particular the method based on Clenshaw-Curtis quadrature. This technique is successful whenever the interval does not contain stationary points. Unfortunately it requires the computation of the inverse of $g$, albeit only at the interpolation points.

Another method for irregular oscillators is proposed by Evans in [8]. Instead of interpolating $f$ by polynomials, we can interpolate $\frac{f(x)}{g^{\prime}(x)}$ using a basis of the form

$$
\sum c_{k} \psi_{k}(g(x))
$$

Then making the transformation $y=g(x)$, as in (1.7), does not require the computation of inverses of $g$. We must, however, be careful in the choice of the basis $\psi_{k}$.

In [5], the problem of solving the acoustic equation was tackled. This method required the computation of oscillatory integrals, for which a new quadrature scheme was derived. At high frequencies, univariate oscillatory integrals are dominated by the contribution from the stationary points and endpoints (multivariate integrals are dominated by contributions from stationary points, resonance points and vertices). Thus we can obtain a high accuracy approximation by numerically integrating near these important points, and throwing away the contributions from the more oscillatory regions. This is accomplished by utilizing smooth windowing functions that focus on $\epsilon$ neighbourhoods of these important points. The part of the integral which we throw away decays exponentially fast as the frequency increases; though the error in the approximation only decays at the same rate as the integral due to quadrature error in each $\epsilon$ neighbourhood. 


\section{Bibliography}

[1] Abramowitz, M. and Stegun, I. A. (1965). Handbook of mathematical functions with formulas, graphs, and mathematical tables (Dover Publications, New York).

[2] Adam, G. and Nobile, A. (2002). Product Integration Rules at ClenshawCurtis and Related Points: A Robust Implementation, IMA Journal of Numerical Analysis 11(2), 271-296.

[3] Alaylioglu, A., Evans, G. and Hyslop, J. (1976). The use of Chebyshev series for the evaluation of oscillatory integrals, The Computer Journal 19(3), 258-267.

[4] Bakhvalov, N. and Vasileva, L. (1968). Evaluation of the integrals of oscillating functions by interpolation at nodes of Gaussian quadratures, USSR Comp. Math. Phys 8, 241-249.

[5] Bruno, O. P. (2003). Fast, high-order, high-frequency integral methods for computational acoustics and electromagnetics, in: Lecture Notes in Computational Science and Engineering 31: Topics in Computational Wave Propagation (Springer, Berlin).

[6] Deaño, A. and Huybrechs, D. (2008). Complex Gaussian quadrature of oscillatory integrals. Technical Report 2008/NA04, University of Cambridge.

[7] Evans, G. (1994). An alternative method for irregular oscillatory integrals over a finite range, International Journal of Computer Mathematics 52(3), 185-193.

[8] Evans, G. (1997). An expansion method for irregular oscillatory integrals, Int. J. Comput. Math. 63(1), 137-148.

[9] Evans, G. A. and Webster, J. R. (1997). A high order, progressive method for the evaluation of irregular oscillatory integrals, Appl. Numer. Math. 23(2), 205-218.

[10] Evans, G. A. and Webster, J. R. (1999). A comparison of some methods for the evaluation of highly oscillatory integrals, J. Comput. Appl. Math. $112(1), 55-69$.

[11] Filon, L. N. G. (1928). On a quadrature formula for trigonometric integrals, Proc. Roy. Soc. Edinburgh 49, 38-47.

[12] Forrey, R. (1997). Computing the Hypergeometric Function, J. Comput. Phys. 137(1), 79-100.

[13] Huybrechs, D. and Olver, S. (2008). Rapid function approximation by modified Fourier series, This volume.

[14] Huybrechs, D. and Vandewalle, S. (2006). On the evaluation of highly oscillatory integrals by analytic continuation, SIAM J. Numer. Anal. 44(3), 1026-1048.

[15] Huybrechs, D. and Vandewalle, S. (2007). The construction of cubature rules for multivariate highly oscillatory integrals, Math. Comp. 76(260), 1955-1980.

[16] Huybrechs, D. and Vandewalle, S. (2007). A sparse discretisation for integral equation formulations of high frequency scattering problems, SIAM J. Sci. Comput. 29(6), 2305-2328.

[17] Iserles, A. (2004). On the numerical quadrature of highly-oscillating integrals I: Fourier transforms, IMA J. Num. Anal. 24(3), 365-391.

[18] Iserles, A. and Nørsett, S. P. (2004). On quadrature methods for highly oscillatory integrals and their implementation, BIT 44(4), 755-772.

[19] Iserles, A. and Nørsett, S. P. (2005). Efficient quadrature of highly oscillatory integrals using derivatives, Proc. R. Soc. Lond. A 461, 1383-1399. 
[20] Iserles, A. and Nørsett, S. P. (2006). From high oscillation to rapid approximation I: Modified Fourier expansions, Technical Report 2006/NA05, University of Cambridge.

[21] Iserles, A. and Nørsett, S. P. (2006). Quadrature methods for multivariate highly oscillatory integrals using derivatives, Math. Comp. 75, 1233-1258.

[22] Kim, K. J., Cools, R. and Ixaru, L. G. (2002). Quadrature rules using first derivatives for oscillatory integrands, J. Comput. Appl. Math. 140(12), 479-497.

[23] Kim, K. J., Cools, R. and Ixaru, L. G. (2003). Extended quadrature rules for oscillatory integrands, Appl. Numer. Math. 46(1), 59-73.

[24] Levin, D. (1982). Procedure for computing one- and two-dimensional integrals of functions with rapid irregular oscillations, Math. Comp. 38(158), 531-538.

[25] Littlewood, R. and Zakian, V. (2003). Numerical Evaluation of Fourier Integrals, IMA Journal of Applied Mathematics 18(3), 331-339.

[26] Lozier, D. and Olver, F. (1994). Numerical Evaluation of Special Functions, Mathematics of Computation, 1943-1993: A Half-century of Computational Mathematics, ed. W. Gautschi (AMS, Providence).

[27] Luke, Y. L. (1954). On the computation of oscillatory integrals, Proc. Cambridge Phil. Soc. 50, 269-277.

[28] Muller, K. (2001). Computing the confluent hypergeometric function, M (a, b, x), Numerische Mathematik 90(1), 179-196.

[29] Olver, F. W. J. (1974). Asymptotics and special functions (Academic Press Inc, New York).

[30] Olver, S. (2007). Moment-free numerical approximation of highly oscillatory integrals with stationary points, Euro. J. Appl. Maths 18, 435-447.

[31] Olver, S. (2006). Moment-free numerical integration of highly oscillatory functions, IMA J. Num. Anal. 26(2), 213-227.

[32] Olver, S. (2006). On the quadrature of multivariate highly oscillatory integrals over non-polytope domains, Numer. Math. 103(4), 643-665.

[33] Patterson, T. (1976). On high precision methods for the evaluation of fourier integrals with finite and infinite limits, Numerische Mathematik 27(1), 41-52.

[34] Piessens, R. and Poleunis, F. (1971). A numerical method for the integration of oscillatory functions, BIT Numerical Mathematics 11(3), 317-327.

[35] Stein, E. M. (1993). Harmonic analysis: Real-variable methods, orthogonality and oscillatory integrals (Princeton University Press, Princeton).

[36] van der Laan, C. and Temme, N. (1984). Calculation of Special Functions: The Gamma Function, the Exponential Integrals and Error-Like Functions, (Centrum voor Wiskunde en Informatica Amsterdam, The Netherlands).

[37] Wong, R. (2001). Asymptotic approximation of integrals (SIAM, Philadelphia).

[38] Zamfirescu, I. (1963). An extension of Gauss's method for the calculation of improper integrals, Acad. RP Romine Stud. Cerc. Mat 14, 615-631. 